\title{
Genetisch bedingte Erythromelalgie im Fokus
}

Fragestellung: Patienten mit der seltenen genetisch bedingten Erythromelalgie mit Mutation im spannungsabhängigen Natriumkanal SCN9A werden detailliert klinisch beschrieben.

Hintergrund: Die genetisch bedingte Erythromelalgie (inherited erythromelalgia, IEM) ist durch dominante "gain-of-function“Mutationen im SCN9A-Gen bedingt, das für den spannungsabhängigen Natriumkanal Nav1. 7 kodiert [1-3]. Sie ist durch anfallsartige Schmerzen und Rötung der Haut charakterisiert und gilt als Modellkrankheit für Schmerz. Trotz vieler Fallbeschreibungen $[4,5]$ fehlte bisher eine systematische Beschreibung des Schmerzphänotyps bei einer gut charakterisierten Kohorte.

Patienten und Methodik: Die Probanden wurden mit Fragebögen, quantitativ sensorischer Testung (QST) nach dem Protokoll des Deutschen Forschungsnetzwerks Neuropathischer Schmerz (DFNS) und Riechtests untersucht und führten über drei Monate ein Schmerztagebuch. Die 26 kodierenden Exone des SCNA9-Gens wurden erneut sequenziert.

Ergebnisse: Sechs Männer und sieben Frauen zwischen 15 bis 77 Jahren nahmen an der Studie teil. Davon gehörten sieben zu einer Familie. Es wurden ins-

McDonnell A, Schulman B, Ali $Z$ et al. Inherited erythromelalgia due to mutations in SCN9A: natural history, clinical phenotype and somatosensory profile. Brain 2016: 139; $1052-65$ gesamt fünf verschiedene Mutationen gefunden. Bei elf der 13 Probanden hatten die Symptome in der ersten Lebensdekade begonnen und waren im Laufe des Lebens gleich geblieben oder milder geworden. Die Zeit bis zur Diagnosestellung betrug im Mittel etwa 20 Jahre. Alle Probanden berichteten Schmerzen und ein Hitzegefühl in den Extremitäten, bei manchen war auch das $\mathrm{Ge}$ sicht betroffen. Schmerzattacken wurden durch Wärme oder Bewegung ausgelöst und durch Kühlen gelindert. Kälte konnte aber auch den Schmerz auslösen oder verstärken. Elf der $13 \mathrm{~Pa}$ tienten hatten zwischen den Attacken geringe Dauerschmerzen. Der Geruchssinn war nicht beeinträchtigt. Angst und Depression waren auf der Hospital Anxiety and Depression Scale (HADS) nur mäßig ausgeprägt, wobei der HADS-Score mit der Stärke der Dauerschmerzen korrelierte. Bei zwei Patienten mit der V400M-Mutation linderte Carbamazepin die Schmerzen, ansonsten zeigte keine Schmerzmedikation Effekte. Die Empfindungsschwellen für Kälte und Wärme waren in betroffenen im Vergleich zu nicht betroffenen Körperarealen deutlich erhöht. Die Schmerzschwellen für Kälte- und Hitzereize waren in nicht betroffenen Körperarealen herabgesetzt. Im Gegensatz zu Trägern anderer Mutationen hatten Träger der F1449V-Mutation besonders hohe Detektionsschwellen für mechanische Reize an nicht vom Schmerz betroffenen Körperstellen.

Schlussfolgerungen: Diskutiert wird, warum die Patienten keine Riechstörung, kaum autonome Symptome und auch geringe Angst- und Depressionswerte haben. Die angehobenen Empfindungsschwellen für Kälte und Wärme wurden als Zeichen einer Small-fiber-Neuropathie bei IEM interpretiert. Die taktile Hypästhesie in klinisch nicht betroffenen Körperarealen war unerwartet und konnte nicht erklärt werden. Die Autoren betonen die Bedeutung der Charakterisierung des Schmerzphänotyps vor dem Einschluss in klinische Studien für neue Analgetika.

\section{- Kommentar von Claudia Sommer, Würzburg}

\section{Gute klinische Übersicht und Datenbank zur IEM}

13 Patienten mit der seltenen Erkrankung der genetisch bedingten Erythromelalgie (IEM) wurden in dieser Studie ausführlich klinisch-algesiologisch charakterisiert. Erstmals liegen somit auch von einer Kohorte von IEM-Patienten QSTDaten vor. Unter dem Aspekt der gefundenen QST-Daten wird auch in Zukunft der Zusammenhang zwischen IEM und Smallfiber-Neuropathie aufzuarbeiten sein [6, 7]. Die vorliegende

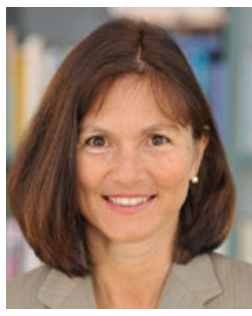

Prof. Dr. med. Claudia Sommer, Würzburg

Leitende Oberärztin, Neurologische Klinik und Poliklinik, Universitätsklinikum Würzburg E-Mail: sommer@uni-wuerzburg.de
Arbeit bietet eine gute Übersicht und Datenbasis zur IEM. Diese Daten sind besonders wertvoll, da es sich bei dieser monogenetischen Schmerzerkrankung um ein Modell für humane neuropathische Schmerzen handelt, von dem Schlussfolgerungen auf Teilaspekte anderer neuropathischer Schmerzerkrankungen gezogen werden können. Zudem zeigt die Fallserie eindrucksvoll, wie sehr sich Phänotypen inter- und intraindividuell trotz zum Teil gleicher Mutation unterscheiden können.

\footnotetext{
Referenzen:

1. Cummins TR et al. J Neurosci 2004; 24: $8232-6$

2. Yang Y et al. J Med Genet 2004; $41: 171-4$

3. Dib-Hajj SD et al. Brain 2005; 128: $1847-54$

4. Fischer TZ et al. Ann Neurol 2009; 65: $733-41$

5. Han C et al. Brain 2009; 132: $1711-22$

6. Faber CG et al. Ann Neurol 2012; 71:26-39

7. Harrer JU et al. Pain 2014; 155: 2199-203
} 